\title{
Piezosurgery in Periodontics
}

\author{
${ }^{1}$ Sakthi Devi, ${ }^{2}$ Mythili Swaminathan, ${ }^{3}$ I Karthikeyan, ${ }^{4} \mathrm{~K}$ Anitha
}

\begin{abstract}
Piezosurgery is a new and innovative method that uses piezoelectric ultrasonic vibrations to perform precise and safe osteotomies. It was first invented by Tomaso Vercelotti to overcome the limitations of traditional instruments in oral bone surgery. Its use was first reported in the field of preprosthetic surgery alveolar crest expansion and sinus grafting. It provides substantial improvement dental/implant surgery, benefiting the surgeon by ease of use and the patient by minimizing surgical trauma and promoting rapid healing. Manual is used to remove small amounts of bone in areas with relatively less dense mineralization. The manual instruments are difficult to control in cortical bone, particularly where precise osteotomies are essential. They are mostly applied for gross cutting of large bone segments. Motor Driven instruments are often used when bone is very dense. Motor driven instruments transform electric or pneumatic energy into mechanical cutting action using the sharpened edge of burs or saw blades. These instruments generate a significant amount of heat in the cutting zone that must be minimized by water irrigation overheating of adjacent tissue may alter or delay the healing response. The reduced rotational speed decreases not only frictional heat but also cutting efficiency. Motorized cutting tools also decrease tactile sensitivity, slower rotational speed necessitates increased manual pressure which increases the macro-vibration of the cutting tool and further diminishes sensitivity. This article discusses about the wide range of application of piezoelectric technique.
\end{abstract}

Keywords: Bone, Osseous surgery, Piezoelectric device, Piezosurgery.

How to cite this article: Devi S, Swaminathan M, Karthikeyan I, Anitha K. Piezosurgery in Periodontics. Int J Prosthodont Restor Dent 2015;5(2):51-55

Source of support: Nil

Conflict of interest: None

\section{INTRODUCTION}

Periodontitis results in significant damage to the tooth supporting bone. Bony deformities occur as a result of this

\footnotetext{
${ }^{1,3}$ Senior Lecturer, ${ }^{2}$ Dean, ${ }^{4}$ Reader

${ }^{1,3,4}$ Department of Periodontics, Indira Gandhi Institute of Dental Sciences, Puducherry, India

${ }^{2}$ Department of Periodontics, Rajah Muthiah Dental College and Hospital, Chidambaram, Tamil Nadu, India
}

Corresponding Author: Sakthi Devi, Senior Lecturer Department of Periodontics, Indira Gandhi Institute of Dental Sciences, Puducherry, India, Phone: 04132615449 e-mail: dr.s.sakthidevi@gmail.com damage, leading to an alteration in normal physiologic bone pattern which needs osseous recontouring. ${ }^{1}$ Traditionally, osseous surgery has been performed by either manual or motor-driven instruments. However, both these methods have their own advantages and disadvantages. ${ }^{2}$ Recently, a novel surgical approach using piezoelectric device has been introduced in the field of periodontology and oral implantology, which promises a safe and effective method in comparison with the conventional technique. The piezosurgery device is used develop positive, physiological architecture of bone support of the involved teeth. Piezoelectric surgery systems use ultrasonic microvibrations to create an osteotomy which makes selective bone cut possible. The piezosurgery device causes little on soft tissue trauma during intraoral bone harvesting ${ }^{3}$ addition surgical access is easier in the posterior region comparison to surgical burs. Since dental surgical techniques have been developed rapidly over the last 2 decades, one surgical technique based on novel application of the principle of ultrasonic vibration has been introduced with wide range of applications in dentistry and periodontics. The purpose of this article is to describe the efficacy and safety of piezoelectric surgery.

\section{INDICATIONS}

In the field of periodontology, it is used in soft tissue debridement to remove the secondary flap after incision through retained periosteum, smoothing of root surfaces by thorough removal of calculus and to regain positive bony margins, bone grafting of an infrabony periodontal defect, ridge augmentation and ridge expansion. In the field of implantology used for implant site preparation, implant removal and bone harvesting and grafting and sinus lifts. In the field of dentistry, it is used for retrograde preparation of root canal, apicectomy, cystectomy, osteogenic distraction tooth extraction, ankylotic tooth extraction and orthodontic surgeries. ${ }^{4}$

\section{CONTRAINDICATIONS}

In certain diseases, such as cardiopathy (heart disorders), uncontrolled diabetes and patient receiving radiotherapies. Also, it should be avoided on patients as well as operator with pacemakers or other implantable electronic devices. An electrosurgical knife could interfere with correct 
functioning of the device. This technique should not be carried on metal or ceramic prosthesis. The ultrasonic vibrations could lead to decementing of such prosthesis. ${ }^{5}$

\section{Advantages}

Piezosurgery requires much less hand pressure, enhanced operator sensitivity and visibility (bloodless), acute tactile sense and control, does not cut soft tissue producing less collateral tissue damage, which results in better healing. The piezosurgery device reduces the frequency of membrane perforation during sinus lift, ${ }^{6}$ reduces patient fear and stress during surgery and causes minimal or no damage to nerves and blood vessels during nerve lateralization procedures because of its ability to cut selectively. ${ }^{7}$

\section{Disadvantages}

It has less control of cutting depth, reduced sensibility and precision due to the vibrations.

Piezosurgery techniques: They were developed in response to the need for greater precision and safety in bone surgery than was available with other manual and motorized instruments.

Micrometric cuts: Maximum surgical precision and intraoperative sensitivity.

Selective cuts: Minimal damage to soft tissue, maximum safety for you and your patients.

Piezosurgery units: It consists of a surgical unit, torque wrench, liquid, peristaltic pump and handpiece. It applies a predefined force to obtain optimium energy transmission as handpiece is attached to torque wrench. A typical piezoelectric handpiece uses a functional frequency between 25 and $29 \mathrm{kHz}$, with the possibility of digital modulation up to $30 \mathrm{kHz}$ as a boost. Piezosurgery devices are fitted with a cooling irrigation system with 0 to $60 \mathrm{ml} / \mathrm{min}$ of variable sterile solution flow. Specific inserts act in a linear vibration pattern, moving over a spatial range between 60 and $210 \mu \mathrm{m}$, at an ultrasonic power Watts gives precise control. ${ }^{8}$ Piezosurgery units are three times more powerful than conventional ultrasonic units (5 Watts), which allows them to cut highly mineralized cortical bone. There are two basic programs for bone and root. In the bone program, it is possible adapt the power to any of four levels, and in the root program, the power can be set either to 'perio' or 'endo' with an automatic feedback system for constant control.

\section{TYPES OF PIEZO INSTRUMENTS}

Sharp instruments: The sharp edge of the instruments enables gentle and effective treatment of the bony structures, such as in osteotomy, implant site pre-paration, osteoplasty techniques and or harvesting bone chips.
Smoothening instruments: The smoothening instruments have diamond surfaces enabling precise and controlled work on the bone structures. Preparing for a sinus window or access to a nerve is possible.

Blunt instruments: Are used for preparing the soft tissue. For elevating schneiders membrane or for lateralizing nerves. In periodontology, these tips are used for root planning.

\section{INSTRUMENTS COLORS}

Gold: For all instruments used to treat bone. The gold color of the instrument is obtained by applying a coating of titanium nitride to improve the surface hardness which means a longer working life.

Steel: For all instruments used to treat soft tissue or delicate surfaces, such as the roots of teeth.

\section{TYPES OF KITS USED}

Basic kit for osteotomy, osteoplasty, extraction, ridge expansion. Sinus lifts kit for preparation of the bone window, separation and elevation of the sinus membrane. ${ }^{9}$

Biological effects on bone cut by a piezoelectric device: Piezoelectric bone surgery appears to be more efficient in the first phase bone healing; it induced an earlier increase in BMP's, controlled the inflammatory process better and stimulated bone remodeling as early as 56 days post-treatment. ${ }^{10}$

\section{STUDIES USING PIEZOSURGERY IN PERIODONTICS}

- Grafting with intraoral bone block is a good way to reconstruct and ever horizontal and vertical bone resorption in future implant sites. ${ }^{11}$

- Membrane perforation rate in this series of 100 consecutive cases using the piezoelectric technique has been reduced with rotary instrumentation to $7 \% .{ }^{12}$

- Piezosurgery proved to be an effective instrument in interventions requiring a significant saving of bone tissue, extreme precision in cutting and respect of soft tissues. $^{13}$

- Piezoelectric surgery in the oral and craniomaxillofacial regard to human subject protection and financial conflicts. ${ }^{14}$

- Piezosurgery prevents brain damage: An experimental study on a new rat model that piezosurgery is a safe method for the performance of osteotomy in close relation to soft tissue including an extremely injury, sensitive tissue, such as brain. ${ }^{15}$

- Sinus floor augmentation with a hydropneumatic technique: A retrospective study: The use of hydropneumatic balloon for the elevation of the sinus membrane is a 
new technique for augmentation procedure. In fact, gradual balloon inflamed invasiveness and atraumatic preparation of the sinus floor membrane is predictable and safe. The relatively short learning curve of this approach for sinus allows for its use in private practice. $^{16}$

- Bone harvesting with piezoelectric device ${ }^{17}$ also suggested that a standard approach to the parietal bone is used either with a bicoronal flap or an incision in the temporoparietal area. An oscillation frequency of 25 to $30 \mathrm{kHz}$ is used to osteotomize the parietal bone.

- Piezosurgical osteotomy for harvesting intraoral block bone graft $^{18}$-a predictable procedure.

- Electric osteotomy in hand surgery as first experience with a new technique suggested a precise procedure. ${ }^{19}$

- Osseous response following resective therapy with piezosurgery; A piezoelectric instrument vibrating in the ultrasonic frequency range was investigated for its potential use in periodontal resective therapy. ${ }^{20,21}$

\section{RIDGE AUGMENTATION}

\section{Studies}

Localized maxillary ridge augmentation using onlay technique with a xenograft block for dental implant placement suggested that Xenograft bone blocks are made from animal derived bone and are available as an 'off the shelf' alternative to autogenous block grafting in attempt to reduce postsurgical morbidity and resorption.

Surgical placement of a $3.5 \times 11 \mathrm{~mm}$ astra tech dental implant (by Dentsply, Mölndal, Sweden) was placed within the prosthetic envelop following the confirmation of full integration of the xenograft block and removal of the fixation screw. It filled the defects around spongiosa blocks and added to the labial contour. ${ }^{22}$

\section{Application of Piezosurgeries in Advanced Periodontology}

The piezosurgery device is used in bone grafting of an infrabony periodontal defect.

Dr Stuart J Forum studied an alternative approach that uses a piezoelectric instrument for the sinus elevation procedure. The membrane perforation rate has been reduced from the average reported rate of $30 \%$ with instrumentation to $7 \%$. Furthermore, all perforations with the piezoelectric technique occurred during the hand instrumentation phase and not with the piezoelectric inserts. ${ }^{23}$ Geraldo prestet et al made a comparative study of two autogenous graft techniques using piezosurgery for sinus lift in shows electric proved 8 weeks as well as 12 weeks after surgery an in growth of vital bone-forming tissue into the osteotomy gap. Schaeren in his recent vitro study has used piezosurgery for transposition of the inferior alveolar nerve in the mandibles of cadaver sheep showed that this technique caused roughening of the epineurium without affecting deeper structures and induced less injury than a conventional rotary bur. ${ }^{24}$

\section{APPLICATIONS IN IMPLANTOLOGY}

Implant site preparation, implant removal and bone grafting and sinus lifts can be done with much ease and less soft tissue trauma. The piezosurgery is a new and modern technique of the bone surgery in implantology.

Selective cutting is possible to different ultrasonic frequencies acting only in hard (tissues) (mineralized) protecting noble anatomical structures with the piezoelectric osteotomy technique.

\section{Studies}

\section{Piezosurgery Applied to Implant Dentistry: Clinical and Biological Aspects}

They transform critical operations in simple, fully executable procedures effectively surgeries performed in different access areas come to be of less risk to soft and neurovascular tissues..$^{25}$

\section{Procedure for Sinus Membrane Elevation}

Sinus augmentation surgery has become well accepted preposthetic procedure for creating sufficient bone volume for the placement of endosseous implants in the atrophic posterior maxilla.

\section{Sinus Membrane Elevation using Piezosurgery}

A total of 35 patients and 40 grafted sinuses were included. The parameters recorded were bony window length and height, bone thickness, osteotomy area, operative time and the number of perforations. Membrane perforations using piezo was minimal. ${ }^{26}$

\section{Corticotomies}

In bone surgery, a corticotomy is a cutting of the bone that may or may not split it into two pieces (bone fracture) but involves cortex only, leaving intact the medullary vessels and periosteum. Corticotomy is particularly important in distraction osteogenesis.

\section{Endoscopically Assisted Tunnel Approach for Minimally Invasive Corticotomies: a Preliminary Report}

The dental community has expressed low acceptance of traditional corticotomy techniques for corticotomyfacilitated orthodontics. These procedures are time 
consuming, entail substantial postoperative morbidity and periodontal risks, and are often perceived as highly invasive.

A total of 114 interdental sites were treated in nine consecutive patients. Under local anesthesia, a tunnel approach requiring one to three vertical incisions per arch (depending on the targeted teeth) was used. Piezosurgical corticotomies and elective bone augmentation procedures were performed underendoscopic assistance. Postoperative cone-beam computerized tomography (CBCT) evaluation was used to confirm adequate corticotomy depth.

Procedures were completed in a mean time of 26 minutes. Follow-up evaluations revealed no loss of tooth vitality, no changes in periodontal probing depth, good preservation of the papillae and no gingival recession. No evidence of crestal bone height reduction or apical root resorption was detected.

The tunnel approach minimizes soft-tissue debridement and permits effective cortical cuts. The combination of piezosurgery technique with endoscopic assistance provides a quick, reliable means to design and perform these corticotomies while maximizing root integrity preservation. Moreover, the sites requiring bone augmentation are selected under direct vision. Compared to traditional corticotomies, this procedure has manifest advantages in surgical time, technical complexity, patient morbidity and periodontium preservation. ${ }^{27}$

\section{SKULL-BASED SURGERY}

\section{Definition}

It is genetic term to denote a specialty of surgery and a group of operations techniques, and approaches to lesions at or involving the base of the skull or its contents.

\section{Studies}

Skull-based surgery involves frequent dissection of the bony skull-based around critical neurovascular structures. Current tools for bone dissection, such as the drill or saw are dangerous as they can cause significant collateral damage to these structures, thus potentially altering functional out comes after skull-based surgery. Piezosurgery, due to its soft tissue sparing and hemostatic effects, enables safe, precise and atraumatic bone dissection with the potential of expanding the capabilities of skull-based surgery and reducing the associated functional morbidity. ${ }^{28}$ Piezosurgery was used to dissect or expose dural and neurovascular structures under nerve monitoring. Operation time, macroscopic preservation, and functional preservation were monitored and correlated with postoperative function. Any complications were correlated with the different phases of surgery and the instruments used.
Operation times were comparable to conventional procedures. Dural integrity was maintained, and the extent of bone removal was increased by piezosurgery. Dissection and exposure of neurovascular structures was achieved with macroscopic preservation and no functional irritation of neural structures. No surgical complications occurred. Piezosurgery increases the extent and radicality of bone removal possible through anatomic and integrity-preserving dissection of critical neurovascular structures in the bony skull base. This enhances exposure and access in various skull base.

\section{CONCLUSION}

The use of piezosurgery devices for dental procedures has numerous advantages when compared with traditional hard and soft tissues methods (motor driven), like absence of the macrovibrations, ease of use, control and safer precise cutting, particularly in complete anatomical areas. Since it has physical and mechanical properties like sparing of vital, neurovascular bundles, better visualization of the surgical field the use of the device and the procedures in the everyday dental practice should be considered.

\section{REFERENCES}

1. Seshan H, et al. Piezosurgery in periodontology and oral implantology. J Indian Soc Periodontol 2009 Sep-Dec; 13(3):155-156.

2. Horton JE, Tarpley TM Jr, Wood LD. The healing of surgical defects in alveolar bone produced with ultrasonic instrumentation, chisel and rotary bur. Oral Surg Oral Med Oral Pathol 1975 Apr;39(4):536-546.

3. Vercellotti T. Piezoelectric surgery in implantology: a case report: a new piezoelectric ridge expansion technique. Int J Periodont Restorat Dent 2000;20(4):358-365.

4. Vercellotti T. Techonological characteristics and clinical indications of piezoelectric bone surgery. Minerva Stomatol 2004;53(5):207-214.

5. Horton JE, Tarpley TM Jr, Jacoway JR. Clinical applications of ultrasonic instrumentation in the surgery removal of bone. Oral Surg Oral Med Oral Pathol 1981 Mar;51(3):236-242.

6. Wallace SS, Mazor Z, Froum SJ, Sang-Choon Cho, Tarnow DP. Schneiderian membrane perforation rate during sinus elevation using piezosurgery: clinical results of 100 consecutive cases. Int J Periodont Restorat Dent 2007 Oct; 27(5):413-419.

7. Beziat JL, Vercellotti T, Gleizal A. What is Piezosurgery? Twoyears experience incraniomaxillofacial surgery. Rev Stomatol Chir Maxillofac 2007;108(2):101-107.

8. Schlee M, et al. Piezosurgery: basic and possibilities. Implant Dentist 2006;15(4):334-338.

9. Vercellotti T, Pollack AS. Piezosurgery: a new device for bone surgery: Part I: sinus grafting and periodontal surgery. Compendium; issue May 2006.

10. Stübinger S, Goethe JW. Bone healing after piezosurgery and its influence on clinical applications. J Oral Maxillofac Surg 2007 Sep.

11. Don-Seck Sohn, et al. Grafting with intraoral bone blocks is good way to reconstruct severe horizontal and vertical. J Periodont Restorat Dent 2007;27(2):127-131. 
12. Wallace SS. Schneideran membrane perforation rate during sinus elevation using piezosurgery. Int J Periodont Restorat Dentist Oct 2007;27(5):413-419.

13. Stomatol M, et al. Retrieval of blade implants with piezosurgery. Minerva Stomatol 2007;56(1-2):53-61.

14. Pitak-Arnnop P, Hemprich A, Sader R, Dhanuthai K, Bertrand JC, Hennig C, et al. Piezoelectric surgery in the oral and craniomaxillofacial region with regard to human subject protection and financial conflicts. Int J Oral Maxillofac Surg 2009;20(4):11321-11341.

15. Pavlíková G, Foltán R, Burian M, Horká E, Adámek S, Hejčl A. Piezosurgery prevents brain damage an experimental study. Int J Oral Maxillofac Surg 2011 Aug; 40(8):840-844.

16. Petruzzi M, et al. Sinus floor augmentation technique with a hyropneumatic technique. Int J Periodont Restorat Dent 2012 April;32(2):205-210.

17. Gonzalez Lagunas J. Calvarial bone harvesting with piezoelectric device. J Craniofac Surg 2007 Nov; 18(6):1395-1396.

18. Lakshmiganthan M, Gokulanathan S. Piezosurgical osteotomy for intra oral bone graft. J Pharm Bioallied Sci 2012 Aug;4(Suppl 2):S165-S168.

19. Hoigre DJ, Stubinger S, Kaenel OV. Piezoelectric osteotomy in hand surgery: first experience with a new technique. BMC Musculoskeletal disorders 2006 April;7:36.
20. Bersani E, et al. Osteoplasty. Int J Periodont Restorat Dentis 2004;24(6):565-577.

21. Bersani E, et al. Osteotomy. Int J Periodont Restorat Dentist 17th May 1993.

22. Hussain I. Localized maxillary ridge augmentation using onlay technique with a xenograft block for dental implant placement: a case series. Smile Den J 2012;7(3):40-45.

23. Schaeren S. Assessment of nerve damage using a novel ultrasonic device for bone cutting. J Oral Maxillofac Surg 2008 Mar;66(3):593-596.

24. Vercellotti T, Dellepiane $M$, et al. Piezoelectric bone surgery in otosclerosis. Acta Otolaryngol 2007 Sep;127(9):932-937.

25. Pereira EC, Grath WC, Noguerira LM, Garcia Junior IR, Okamoto R. Piezosurgery applied to implant dentistry: clinical and microbiological aspects. J Oral Implant 2014 July; 40(SI):401-408.

26. Nevins M, Vercelloti T. Sinus membrane elevation using piezosurgery. Int J Periodont Restorat Dent 2000;20(4):359-365.

27. Hernandez-Alfaro F, et al. Endoscopically assisted tunnel approach for minimally invasive corticotomies: a preliminary report. J Periodont 2012;83:574-580.

28. Jayawardene D, Choy J, Kuthubutheen G, Rajan P. Piezosurgery applications in skull base surgery. J Neurol Surg B 2012;73-A: 429. 